\title{
Comparative study of antioxidants as cancer preventives through inhibition of HIF-1 alpha activity
}

\author{
Jyoti Upadhyay, Rajesh K. Kesharwani, Krishna Misra* \\ Indian Institute of Information Technology, Allahabad, India-211002; Krishna Misra - Email: krishnamisra@hotmail.com; *Corresponding \\ author
}

\begin{abstract}
: be designed to validate these observations.

\section{Background:}

One of the important factors highlighted recently in cancer is the activation of glycolysis leading to angiogenesis [1] and cell proliferation, decreased apoptosis, cellular immortalization and invasion/metastasis. Novel molecules for cell proliferation are provided through glycolysis, which is activated by the well known factor HIF-1 $\alpha$ [2-4]. Transcription factor is a guide to many cancers by activating the transcription of many genes that code for proteins involved in several pathways which are closely related to cancer growth. The progressive survival of cancer cells depends on glycolytic energy through ATP generation [5-6] and the level of ATP is reduced remarkably under oxygen starved conditions in the absence of HIF-1 $\alpha$ [2]. In most common human cancers as well as in pre-neoplastic and pre-malignant lesions, such as colonic adenoma, breast ductal carcinoma HIF-1 $\alpha$ is over-expressed. It is reported that HIF-1 $\alpha$ expression may occur very early in carcinogenesis, before histological evidence of angiogenesis or invasion [3]. It has been suggested that HIF-1 $\alpha$ is a biomarker of carcinogenesis and for drug design, pharmaceutical companies use it as suitable target for cancer chemoprevention.
\end{abstract}

Received May 15, 2009; Revised June 18, 2009; Accepted November 15, 2009; Published December 06, 2009

HIF-1 $\alpha$ (hypoxia inducible factor-1 $\alpha$ isoform) has been exploited as a target in cancer therapeutics. HIF-1 $\alpha$ is the isoform-2 of HIF-1 $\alpha$ subunit. It is a 735 residues long protein modeled in this study. The HIF-1 $\alpha$ is absolutely critical for continued survival of cancer cells as it is involved in the activation of glycolysis and it helps an oxygen-starved cell convert sugar to energy without using oxygen. It also initiates angiogenesis to bring in a fresh oxygen supply. HIF-1 $\alpha$ operates only in presence of free radicals. In the present study, five antioxidants, namely lycopene, ascorbic acid, $\alpha$-tocopherol, curcumin and curcumin dipiperoyl ester which are potent scavengers of reactive oxygen species (ROS) have been docked to HIF-1 $\alpha$ modeled protein in order to assess their binding and consequently, their inhibitory activity. The binding energy score has been found to be in the order, curumin dipiperoyl ester $>$ lycopene $>$ curcumin $>$ tocopherol $>$ ascorbic acid. However, subsequent experiments should

Studies suggest that HIF-1 $\alpha$ can only operate in the presence of free radicals and it does not work if antioxidants remove these free radicals [7]. They showed that while this protein was abundant in untreated cancer cells taken from mice, it disappeared in vitamin C-treated cells. It might be possible that antioxidants adopted two alternative pathways for reducing the activity of HIF-1 $\alpha$, firstly, these directly remove the free radicals and make the survival of HIF- $1 \alpha$ difficult and another way may be that these antioxidants bind at the active site of the protein to inhibit its activity. HIF1 is a hetero-dimer composed of two subunits (an alpha and a beta). The beta subunit has been recognized as the aryl hydrocarbon receptor nuclear trans-locator (ARNT) [9]. Two alternative transcripts of hypoxia inducible factor-1gene encodes for different isoforms (NP 001521.1 and NP 851397.1). The HIF1 alpha is isoform 2 (NP_851397.1) and it is shorter with a distinct Cterminus, compare to isoform 1 as it lacks an alternate segment at the $3^{\prime}$ CDS region. Therefore, it is important to describe the mechanism of in induction by HIF-1 $\alpha$. However, the protein databank (PDB) contains the NMR structure of cysteine/histidine-rich $1(\mathrm{CH} 1)$ domain of p300 bound to the C-terminal transactivation domain of HIF-1 $\alpha$, which is smaller than the target receptor protein [8]. Hence, we employed threading technique to construct the model. We then used docking tools to evaluate the binding of anti-oxidants like lycopene, curcumin, $\alpha$ tocopherol and ascorbic acid. We also docked curcumin dipiperoyl ester for comparison as its bioavailability is enhanced remarkably to curcumin and it is a potent anticancer drug to curcumin [10].

\section{Methodology:}

Target sequence:

The 735 residue long protein sequence of Human hypoxia inducible factor 1 (NCBI_ID: NP_851397.1 (hypoxia-inducible factor 1, alpha subunit isoform 2) was downloaded from NCBI RefSeq database [12].

\section{BLAST search:}

The target sequence for HIF-1 alpha was submitted to NCBI-Blast and searched against PDB [13] to select suitable structural templates.

\section{Wurst server:}

Wurst server is used in the absence of a suitable homolog. The target sequence is aligned to more than 3000 templates using a dynamic programming algorithm with a structure and sequence features based score function [14].

\section{ArchPRED:}

We used ArchPRED for loop region prediction [15].

\section{SwissPdbViewer:}

We used swisspdbviewer to subsequently model the structure using the selected template and loop structures [16].

\section{RAMPAGE server:}

The RAMPAGE server is used to validate the predicted model [17].

\section{Discussion:}

Ramachandran plot analysis of the modelled structure show $77.4 \%$ in favoured region, $14.1 \%$ in allowed region and $8.1 \%$ in outlier region of the plot. This modeled protein structure has five cavities detected using the molegro software with volumes $288.768 \mathrm{~A}^{3}, 29.184 \mathrm{~A}^{3}$, $26.112 \mathrm{~A}^{3}, 25.6 \mathrm{~A}^{3}$ and $19.968 \mathrm{~A}^{3}$. The cavity with volume 288.768 $\mathrm{A}^{3}$ shows good energy score for all the five antioxidants (lycopene, ascorbic acid, tocopherol, curcumin and dipiperoyl ester of curcumin) used in this study. The ranking of the anti-oxidants using the energy score is curcumin-dipiperoyl ester \& lycopene < curcumin « tocopherol < ascorbic acid. Data shows curcumin dipiperoyl ester with the lowest energy score is most favourable. The five antioxidants used in the study exhibit hydrogen bond interaction with the interacting residues as shown in Figures 1. The analysis shows ascorbic acid with maximum $\mathrm{H}-$ bond interaction and tocopherol with minimum $\mathrm{H}$-bond interactions. Table 1 (see supplementary material) shows the interacting properties of the anti-oxidants to the target protein. Curcumin also show low energy score which is close to lycopene. The energy score of curcumin molecule was changed by substituting it with dipiperoyl ester as reported eslwehere [11]. 


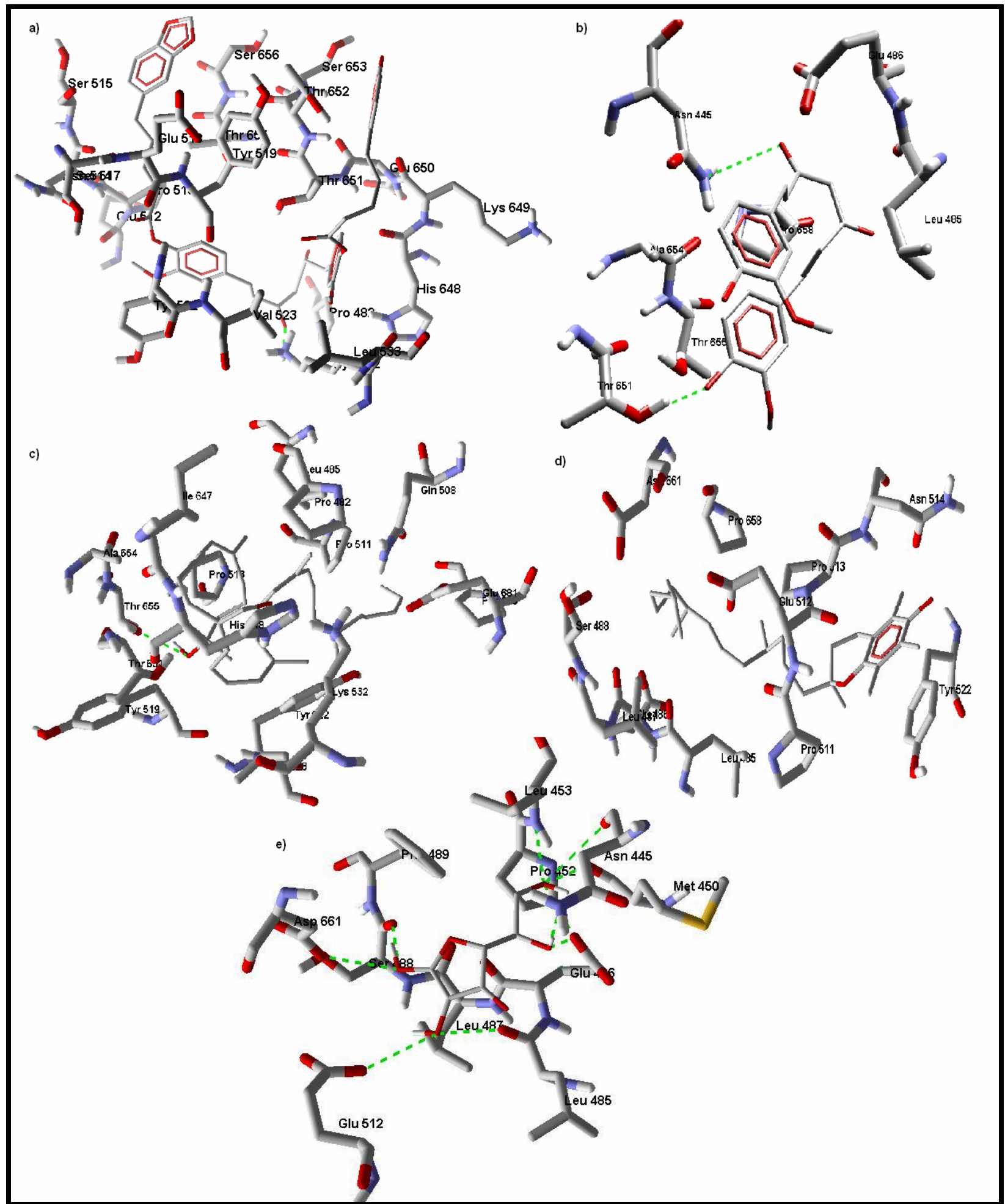

Figure 1: HIF-1 $\alpha$ protein docked with (a) curcumin dipiperoyl ester; (b) curcumin; (c) lycopene; (d) tocopherol; (e) ascorbic acid

\section{Conclusion:}

It is known that antioxidants inhibit the activity of HIF-1 $\alpha$ by scavenging free radicals. Data here show that the five antioxidants (lycopene, ascorbic acid, $\alpha$ - tocopherol, curcumin and curcumin conjugates with piperic acid) have similar mode of binding at the site of the hypoxia inducing factor-1 $\alpha$ with varying binding energy scores. Current analysis also shows the H-bonds interactions of these compounds with the active sites. However, subsequent experiments should be designed to validate these observations. 


\section{Bioinformation}

Reference:

[1] RA Gatenby, RJ Gillies, Nat Rev Cancer. 4: 891 (2004)

[2] AL Harris. Nat Rev Cancer. 2: 38 (2002)

[3] CJ Schofield, PJ Ratcliffe. Nat Rev Mol Cell Biol. 5: 343 (2004)

[4] RH Xu et al., Cancer Res. 65: 613 (2005)

[5] A Ramanathan et al, Proc Natl Acad Sci U S A. 102: 5992 (2005)

[6] GL Semenza Nat Rev Cancer 3: 721 (2003)

[7] P Gao et al., Cancer Cell 12: 230 (2007)

[8] SJ Freedman et al., Proc Natl Acad Sci USA. 99: 5367 (2002)

[9] G Höpfl et al., Am J Physiol Regul Integr Comp Physiol. 286: R608 (2004)
[10] S Mishra et al., Bioorganic \& Medicinal Chemistry 13: 1477 (2005)

[11] S Mishra et al., Free Radical Biology and Medicine 38: 1353 (2005)

[12] KD Pruitt et al., Nucleic Acids Res. 35: D61 (2007)

[13] F Stephen et al., Nucleic Acids Res. 25: 3389 (1997)

[14] AE Torda et al., Nucleic Acids Res. 32: 532 (2004)

[15] N Fernandez-Fuentes et al., Nucleic Acids Res. 34: 173 (2006)

[16] N Guex, MC Peitsch, Protein Data Bank Quarterly Newsletter 77: 7 (1996)

[17] SC Lovell et al., Proteins: Structure, Function \& Genetics 50: 437 (2002)

Edited by P. Kangueane Citation: Upadhyay et al., Bioinformation 4(6): 233-236 (2009) License statement: This is an open-access article, which permits unrestricted use, distribution, and reproduction in any medium, for noncommercial purposes, provided the original author and source are credited. 


\section{Bioinformation}

www.bioinformation.net

Hypothesis

\section{Supplementary material:}

Table1: Comparative binding Energy Scores of the five antioxidants

\begin{tabular}{llll}
\hline Antioxidants & $\begin{array}{l}\text { MolDock Score } \\
\text { (Energy Score) }\end{array}$ & $\begin{array}{l}\text { H-bond interaction } \\
\text { energy }\end{array}$ & Common interacting residues \\
\hline Dipiperoyl ester of curcumin & -200.299 & -3.393 & Pro 482, Glu 512, Pro 513, Tyr 522, Thr 651, Thr 655 \\
Lycopene & -182.405 & -2.5 & Pro 482, Leu485, Pro513, Tyr522, Thr651, Thr655 \\
Curcumin & -151.557 & -3.8 & Leu485, Glu486, Thr651, Thr655 \\
Tocopherol & -128.9 & 0.27 & Leu485, Glu486, Glu512, Pro 513, Tyr 522 \\
Ascorbic acid & -94.5 & -19.4 & Leu485, Glu486, Glu512 \\
\hline
\end{tabular}

